\title{
ENSAYOS
}




\section{LA EDUCACION FRANCESA: IDEAS PARA UNA REFORMA*}

Pierre Bourdieu

\section{Exposición de motivos}

La cuestión de los contenidos y de los fines de la enseñanza no puede contentarse con respuestas generales pero vagas, y apropiadas para lograr la unanimidad a buen precio: nadie en efecto podría impugnar que toda enseñanza debe formar espíritus abiertos, dotados de disposiciones y de saberes necesarios para adquirir sin cesar nuevos saberes y adaptarse a situaciones siempre renovadas. Esta intención universal pide, a cada momento, determinaciones particulares: en función, hoy en día, de los cambios de la ciencia, que no deja de redefinir la representación del mundo natural y del mundo social; en función también de las transformaciones del entorno económico y social, especialmente de los cambios que han afectado el mercado de trabajo debido a las innovaciones tecnológicas y a las reestructuraciones de las empresas industriales, comerciales y agrícolas. De todas estas transformaciones, las que más directamente se relacionan con el sistema de enseñanza son sin duda el desarrollo de los medios de comunicación modernos (en particular la televisión), capaces de competir con la acción escolar 0 de contrarrestarla, y también las modificaciones profundas del papel que incumbe, sobre todo en el orden ético, a instancias pedagógicas como la familia, el sitio de trabajo, las comunidades de los pueblos o de los barrios y las iglesias.

También es necesario tomar nota de las transformaciones del sistema de enseñanza mismo, evitando adoptar, al evocarlas, el lenguaje apocalíptico de la crisis o, aún peor, el tono de condenación profética que busca sus víctimas propiciatorias en el cuerpo docente o sus órganos representativos. En grados diferentes según los sectores y los niveles, las relaciones sociales que son constitutivas de la institución educativa -relación entre maestros y alumnos, relación entre padres de familia y maestros, relación entre maestros de diferentes generaciones - se han transformado profundamente bajo el efecto de factores sociales tales como la urbanización, la prolongación general de la escolaridad y la transformación de la relación entre el sistema escolar y el mercado de trabajo, teniendo como consecuencia una verdadera decepción colectiva a propósito de la escuela.

El sentimiento de desconcierto o de rebelión que suscitan estos cambios resulta, por una parte, del hecho de que ellos no han sido ni pensados ni queridos como tales: el cuestionamiento, o más o menos consciente del contrato tácito de delegación que une una sociedad a su escuela, deja, en los cimientos mismos del sistema de enseñanza, una especie de vacío, generador de angustia. Para conjurar las tentaciones regresivas que el sentimiento de crisis refuerza tanto en los maestros como en los alumnos y padres de familia, es necesario tratar de repensar los principios sobre los cuales puede edificarse un sistema de enseñanza tan democrático como sea posible al mismo tiempo que adaptado a las exigencias del presente y capaz de responder a los desafíos del porvenir.

\footnotetext{
* El 27 de marzo de 1985. Pierre Bourdieu envió al presidente Mitterrand, en nombre del Colegio de Francia, el documento que ofrecemos en versión española y que el mandatario francés había solicitado a aquella institución. El texto original fue publicado en Le Monde de l'Education (Mai, 1985). La presente traducción española es de Rubén Sierra Mejía.
} 
Una reflexión sobre los fines de la escuela no puede ignorar las contradicciones inscritas en una institución consagrada a servir a intereses diferentes, incluso antagónicos. Estas contradicciones, que los límites de la acción propiamente escolar hacen aparecer a menudo como antinomias insuperables ("democratización "/"selección", "cuantitativo"/"cualitativo", "público"/"privado", etc.), disponibles para todas las exploraciones polémicas y políticas, continúan presentes en el esfuerzo mismo por superar las tensiones entre exigencias opuestas o entre los fines propuestos y los medios indispensables para alcanzarlos.

Se puede impugnar a un individuo o a un grupo, cualesquiera que ellos sean, el derecho a legislar en estas materias y a sustituir en esta forma al conjunto de grupos que pretenden examinar las orientaciones del sistema de enseñanza para hacer valer allí sus intereses. Es poco probable que un programa educativo pueda obtener el asentimiento general. Por lo demás, en el estado actual de las instituciones de enseñanza, de hecho se lo cuestiona en lo que concierne a este asunto esencial. Por eso el enunciado implícito de un conjunto coherente de principios directrices tiene al menos por virtud obligar a poner en cuestión y en discusión los presupuestos, o los prejuicios, que son fundamento incierto, por indiscutivo, de políticas escolares. Parece además que muy a menudo hay acuerdo entre los imperativos técnicos que tienen por objetivo asegurar el progreso y la enseñanza de las ciencias y los imperativos éticos incluidos en la idea misma de una sociedad democrática.

De tal suerte que se puede ir más lejos en la definición de una enseñanza a la vez más racional y más justa sin enfrentar los problemas que dividen a menudo a los usuarios de la escuela o sus portavoces.

Este texto no quiere ser ni un plan ni un proyecto de reforma. Es el producto, modesto y provisional, de una reflexión. Sus autores, comprometidos con la investigación y con la enseñanza de la investigación, tienen conciencia de estar alejados de las realidades más ingratas de la enseñanza; pero quizás, por eso mismo, estén ellos liberados de posiciones previas y de objetivos a corto término.

\section{Los principios}

\section{La unidad de la ciencia y la pluralidad de las culturas}

Una enseñanza armónica debe poder conciliar el universalismo inherente al pensamiento científico y el relativismo de que tratan las ciencias humanas, atentas a la pluralidad de los modos de vida, a las sabidurías y a las sensibilidades culturales.

Imponiéndose ella misma el veto de definirse por relación a tal o cual orientación moral, la escuela no puede sustraerse a las responsabilidades éticas que inevitablemente le incumben. Por esta razón uno de sus objetivos mayores podría ser inculcar las disposiciones críticas que enseñan las ciencias de la naturaleza y las ciencias del hombre. En esta perspectiva, la historia de las ciencias y de las obras culturales, enseñadas en la forma apropiada a cada nivel, debería proporcionar antídotos contra las formas antiguas o nuevas de irracionalismo o de fanatismo de la razón. Asimismo, las ciencias sociales deberían conducir a un juicio ilustrado sobre el mundo social y ofrecer armas contra las manipulaciones de toda naturaleza. Muy formadores serían, por ejemplo, el examen crítico del funcionamiento y de las funciones de los sondeos de opinión o el desmonte de los mecanismos de delegación, apoyado en la historia de las instituciones políticas. 
Entre las funciones impartidas a la cultura, una de las más importantes es sin duda el papel de técnica de defensa contra todas las formas de presión ideológica, política o religiosa: este instrumento de pensamiento libre, a la manera de las artes marciales en otros terrenos, puede permitir al ciudadano de hoy en día protegerse contra los abusos de poder simbólico, del cual él es objeto -aquellos de la publicidad, de la propaganda y del fanatismo político y religioso.

Esta orientación pedagógica tendría por fin desarrollar un reparto sin fetichismo de la ciencia como forma acabada de la actividad racional, al mismo tiempo que una vigilancia armada contra ciertos usos de la actividad científica y sus productos. No se trata de fundar una moral sobre la ciencia, real o idealizada, sino de transmitir una actitud crítica respecto de la ciencia y de sus usos, que se desprenda de la ciencia misma o del conocimiento de los usos que se hacen de ella.

El único fundamento universal que puede dársele a una cultura reside en el reconocimiento de la parte de arbitrariedad que debe ella a su carácter histórico: se tratará entonces de poner en evidencia esta arbitrariedad y de elaborar los instrumentos necesarios (aquellos que proveen la filosofía, la filología, la etnología, la historia o la sociología) para comprender y aceptar otras formas de cultura; de allí la necesidad de recordar el enraizamiento histórico de todas las obras culturales, comprendidas entre éstas las obras científicas. Entre las funciones posibles de la cultura histórica (integración nacional, comprensión del mundo presente, reapropiación de la génesis de la ciencia), una de las más importantes, desde ese punto de vista, es la contribución que puede aportar ella al aprendizaje de la tolerancia a través del descubrimiento de la diferencia, pero también de la solidaridad entre las civilizaciones.

Las razones propiamente científicas, especialmente los progresos asegurados por el método comparativo, se conjugan con las razones sociales —en particular las transformaciones de un mundo social en donde hombres que pertenecen a diferentes tradiciones se encuentran cada vez más a menudo conducidos a comunicarse o a cohabitar debido a la extensión de los movimientos migratorios- para imponer la apertura de la enseñanza al conjunto de civilizaciones históricas y a las grandes religiones, consideradas a la vez en su coherencia interna y en las condiciones sociales de su emergencia y de su desarrollo. Pero para hacerlo sin sobrecargar en exceso los programas, importa ante todo romper con la visión etnocéntrica de la historia de la humanidad que hace de Europa el origen de todos los descubrimientos y de todos los progresos; de introducir, desde la escuela primaria, elementos de cultura geográfica y etnográfica apropiados para acostumbrar al niño a admitir la diversidad de usos (en materia de técnicas del cuerpo, de vestidos, de habitación, de alimentación...) y de sistemas de pensamiento; de hacer aparecer, especialmente en la enseñanza de la historia, de las lenguas y de la geografía, la mezcla de necesidad ecológica o económica y de arbitrariedad social que caracterizan las elecciones propias de las diferentes civilizaciones; todo esto recordando los innumerables préstamos de técnicas e instrumentos a través de los cuales se han constituido las diferentes civilizaciones, comenzando por la nuestra.

La enseñanza debería en esta forma reunir el universalismo de la razón, que es inherente a la intención científica, y el relativismo que enseñan las ciencias históricas, atentas a la pluralidad de sabidurías y de sensibilidades culturales. No se puede conciliar la confianza en la unidad de la razón científica y la conciencia de la pluralidad de razones culturales más que a condición de fortalecer la flexibilidad y la adaptabilidad cognoscitivas 
que se adquieren en la confrontación constante del pensamiento con los universos indefinidamente variados y sin cesar renovados de la naturaleza y de la historia.

\section{La diversidad de las formas de excelencia}

La enseñanza debería toda ponerse en ejecución para combatir la visión monista de la "inteligencia" que conduce a jerarquizar las formas de realización en relación con una de ellas, y debería multiplicar las formas de excelencia cultural socialmente reconocidas.

Si el sistema escolar no tiene el dominio completo de la jerarquía de competencias que él garantiza, puesto que el valor de las diferentes formaciones depende fuertemente del valor de los puestos a los cuales da acceso, el efecto de consagración que él ejerce no es por lo tanto despreciable: trabajar para debilitar o abolir las jerarquías entre las diferentes formas de aptitud, tanto en funcionamiento institucional (los coeficientes, por ejemplo) como en el espíritu de los maestros y de los alumnos, seria uno de los medios más eficaces (en los límites del sistema de enseñanza) de contribuir al debilitamiento de las jerarquías puramente sociales. Uno de los vicios más escandalosos del sistema actual reside en el hecho de que él tiende cada vez más a no conocer y no reconocer sino una única forma de excelencia intelectual, aquella que representa la sección (C- (o S) de los liceos y su prolongación en las grandes escuelas científicas. Por el privilegio cada vez más absoluto que él confiere a cierta técnica matemática, tratada como instrumento de selección o eliminación, tiende a hacer aparecer todas las demás formas de competencia corno inferiores; los detentadores de estas competencias mutiladas están así condenados a una experiencia más o menos desafortunada tanto en la cultura que ha recibido como de la cultura escolar-mente dominante (esto es, sin duda, uno de los orígenes del irracionalismo que florece actualmente). En cuanto a los detentadores de la cultura socialmente considerada como superior, ellos están cada vez más a menudo condenados, salvo un esfuerzo excepcional y condiciones sociales muy favorables, a la especialización prematura, con todas las mutilaciones que la acompañan.

Por razones inseparablemente científicas y sociales, habrá que combatir todas las formas, incluso las más sutiles, de jerarquización de las prácticas y de los saberes especialmente aquellas que se establecen entre lo "puro" y lo "aplicado", entre lo "teórico" y lo "práctico" o "técnico", y que en la tradición escolar francesa asumen una fuerza particular-, al mismo tiempo que habrá que imponer el reconocimiento social de una multiplicidad de jerarquías de competencia distintas e irreductibles.

El sistema de enseñanza y la investigación son víctimas, en todos los niveles, de los efectos de esta división jerárquica entre lo "puro" y lo "aplicado' que se establece entre las disciplinas y el seno de cada disciplina, y que es una forma transformación de la jerarquía social de lo "intelectual"y lo "manual". De ello resultan dos perversiones que se tratan de combatir metódicamente por medio de una acción sobre las instituciones y sobre los espíritus: en primer lugar, la tendencia al formalismo que desanima a ciertos espíritus; en segundo lugar, la desvalorización de saberes concretos, de manipulaciones prácticas y de la inteligencia práctica que le está asociada. Una enseñanza armónica debería realizar un justo equilibrio entre el ejercicio de la lógica racional por medio del aprendizaje de un instrumento de pensamiento como las matemáticas y la práctica del método experimental, sin olvidar todas las formas de la destreza manual y la habilidad corporal. El acento podría ponerse sobre las formas generales de pensamiento por las cuales se han constituido, a lo largo de los siglos, las ciencias y las técnicas. Sí las matemáticas nacieron en Grecia, nuestra ciencia no ha podido constituirse verdaderamente sino dos mil años más tarde, en un tejido en el que la urdimbre sería la teoría, a menudo de tipo matemático, y la trama la 
experimentación, gracias a un vaivén constante de la hipótesis téorica a la experimentación que la infirma o la confirma. Encerrando lo real en una red de observaciones o de experimentaciones privilegiadas, la ciencia ha permitido la conquista de especies de verdad aproximada, cuyo grado de aproximación cada vez más grande puede él mismo ser evaluado gracias al cálculo de errores o al cálculo de probabilidades aplicados a la noción misma de medida. La vigilancia crítica sobre los límites de validez de las operaciones y de los resultados de la ciencia, se impone particularmente en un mundo en que intervienen incesantemente porcentajes y probabilidades: son raros los ciudadanos que conciben claramente lo que son las consecuencias, al cabo de diez años, de un alza (o de una baja) del 10/o en un índice económico y que tienen conciencia del carácter artificial, pero útil, de un índice semejante, fundado sobre bases estadísticas. Sin embargo, muchas de nuestras decisiones corrientes reposan, lo más a menudo sin saberlo, sobre tales bases: por ejemplo, un automóvil determinado no es en sí superior a tal competidor comparable, pero en los cientos de miles de automóviles de este modelo, podemos encontrar elementos para evaluar las oportunidades que tengan; meteorología, localmente, no puede evaluar más que posibilidades de lluvia, y es así como describe sus pronósticos la prensa de los Estados Unidos. Parece pues importante que a través de una iniciación que se puede emprender desde la escolaridad obligatoria, aprenda cada uno, al menos intuitivamente, a calcular los riesgos.

Dándole su justo lugar a la teoría que, en su definición exacta, no se identifica ni con el formalismo ni con el verbalismo, y a los métodos lógicos de razonamiento que, en su mismo rigor, contienen una extraordinaria eficacia heurística, la enseñanza debe darse por fin, en todos sus dominios, realizar productos y poner al aprendiz en posición de descubrir por sí mismo. Se puede producir una "manipulación" de química o de física en lugar de recibirla completamente montada y registrar los resultados; se puede producir una pieza de cine, el informe sobre una obra (preferencial-mente con la intención de un verdadero diario de alumnos o de estudiantes) o aún una carta a la seguridad social, unas instrucciones de empleo o un acta de accidente, en lugar de sólo disertar; esto sin olvidar que hay lugar para el descubrimiento activo en las actividades teóricas, como las lógicas o las matemáticas. Con este espíritu, la enseñanza artística concebida como enseñanza profunda de una de las prácticas artísticas (música o pintura o cine, etc.), libre y voluntariamente elegida (en lugar de ser, como hoy en día, indirectamente impuesta), recuperará un lugar eminente. En este dominio, más que en cualquier otra parte, habrá que subordinar el discurso a la práctica (la de un instrumento, incluso de la composición, del dibujo o de la pintura, de la disposición del marco de vida, etc.). La revocación de las jerarquías también deberían conducir, sobre todo en los niveles elementales, a enseñar, tanto como las bellas artes, las artes aplicadas, de gran utilidad en la existencia cotidiana, como las artes gráficas, el arte de editar o de la publicidad, las artes audiovisuales, la fotografía.

\section{La multiplicación de las oportunidades}

Sería importante atenuar, tanto como sea posible, las consecuencias del veredicto escolar e impedir que los éxitos tengan un efecto de consagración y los fracasos un efecto de condenación vitalicia, multiplicando las ramificaciones y los pasos entre las ramificaciones y debilitando todos los cortes irreversibles.

Deberían ponerse en ejecución todos los medios para atenuar los efectos negativos de los veredictos escolares que obran como profecías auto-confirmadoras: se trataría de minimizar el efecto de consagración cuando fomenta una seguridad estatutaria, y sobre todo el efecto de estigmatización que abarca a las víctimas socialmente señaladas de 
veredictos escolares en el círculo vicioso del fracaso. Las sanciones negativas, sobre todo cuando se aplican a adolescentes que, más que en cualquier otra edad, están afrontados a la cuestión de su identidad y especialmente expuestos a crisis más o menos dramáticas, pueden condenar al desánimo, a la dimisión, incluso al desespero. Velar por reducir los efectos incontrolados de todos los veredictos contribuirá sin duda a reducir la ansiedad con respecto a la escuela que no deja de desarrollarse, tanto en los padres como en los hijos, con toda suerte de consecuencias sicológicas, incluso sicopatológicas, y sociales.

Lo que no significa que se deba resolver el problema de la "selección por el fracaso", como se dice a veces, por medio de un rechazo de selección que conduce a diferir siempre el momento de la verdad, con toda suerte de consecuencias funestas, tanto para los individuos a que concierne como para la institución. Conceder un derecho ficticio de entrada, es exponer a hacer pagar muy caro a los individuos y a toda la institución las consecuencias de un mal comienzo. No se puede hacer trampas con la lógica real del aprendizaje y sólo se les debe asegurar a todos, aunque fuese al precio de un esfuerzo especial, un buen comienzo. Se trata de tornar todas las medidas propias para dar a los más desprovistos de buenas condiciones de formación y contrarrestar todos los mecanismos que conducen a colocarlos en las peores condiciones (como la extraña lógica que somete a clases más difíciles los maestros debutantes o maestros auxiliares mal formados, mal pagados y sobrecargados de cursos). Es claro, en efecto, que no se podría esperar una especie de tratamiento sicosociológico que haga desaparecer por milagro fracasos que sólo se puede esperar reducir realmente más que al precio de un aumento del número de maestros y sobre todo de una mejoría de sus condiciones de formación y de trabajo: se conocen, en efecto, las carencias extremas de que sufre la enseñanza francesa, particularmente a nivel superior, en todo lo que concierne a la infraestructura específica de la vida intelectual -bibliotecas ( de las que aquí no se repetirá sus escandalosas insuficiencias), instrumentos de trabajo, tales como manuales, colecciones de textos de calidad, traducciones científicas, bancos de datos, etc.

Dicho esto, maestros más conscientes de la pluralidad de formas de excelencia, por tanto de los límites de validez de sus juicios y efectos traumatizantes que pueden ejercer, se los debería conducir a que eviten todos los veredictos sin apelación sobre las capacidades de sus alumnos globalmente caracterizados, y a que tengan siempre en mente que, en todos los casos, juzgan una realización exacta y parcial, y no una persona tomada en su esencia y naturaleza. La pluralidad de formas de éxito reconocidas, que liberaría a los maestros de la obligación de moderar y evaluar todos los espíritus según un único modelo, unida a la pluralidad de pedagogías que permitiría valorizar y exigir resultados diferentes (en los límites del mínimum cultural común exigible en cada nivel), podría hacer de la escuela no un lugar de fracaso y estigmatización para los más desfavorecidos social-mente, sino un lugar en el que todos podrían y deberían encontrar su manera propia de triunfar.

Para que la evaluación necesaria de las aptitudes tome la forma de un consejo de orientación más que de un veredicto de exclusión, habría que multiplicar las ramificaciones socialmente equivalentes (por oposición a las carreras jerarquizadas de hoy en día). Todas las facilidades institucionales se les deberían dar a quienes quisieran pasar de una ramificación a otra o combinar aprendizajes asociados a ramificaciones diferentes. La rigidez de las trayectorias obligadas, de los cursos irreversibles, que da un peso casi fatal a las escogencias iniciales y a los veredictos escolares de exclusión, se la debería combatir por todos los medios. La necesaria consideración de las diferencias (en las capacidades y los ritmos de adquisición o las formas de espíritu) y la orientación de los alumnos hacia ramificaciones diferentes, deberían acompañarse de medidas que tiendan 
concretamente — suministrando, por ejemplo, buenos docentes y equipos de calidad- a revalorizar las ramificaciones que las jerarquías vigentes (en los espíritus y en el entorno social) llevan a considerar inferiores. El curso tomaría la forma de una especialización progresiva por la orientación hacia establecimientos pluridisciplinarios y abren el acceso a establecimientos más especializados. Sería necesario que antes de decidir sobre la selección de su especialidad, los jóvenes puedan hacer cursos preparatorios en establecimientos diversificados.

Sería necesario también trabajar por destruir o reducir la tendencia a sacralizar el título escolar, especie de esencia social escolarmente garantizada que cumple en nuestras sociedades una función completamente semejante a la del título nobiliario en otras épocas, impidiendo a unos rebajarse en el cumplimiento de ciertas tareas juzgadas indignas, a otros aspirar a porvenires vedados. Importará por esto revalorizar realizaciones efectivas: por ejemplo, introduciendo en todos los reclutamientos un contingente de elecciones y de promociones sobre trabajos efectuados y realizaciones reales (como, en el caso particular de los profesores, la introducción de innovaciones pedagógicas ejemplares o una consagración excepcional). Una de las taras más graves del sistema burocrático francés reside en el hecho de que un incapaz escolarmente garantizado y un competente escolar-mente desprovisto están separados de por vida, tales el plebeyo y el noble, en todos los puntos de vista socialmente pertinentes. La selección por medio del diploma no es perjudicial más que en la medida en que continúa actuando, mucho más allá de su meta, durante toda la duración de la carrera, siendo la evaluación real del trabajo realizado injustamente sacrificada a los intereses corporativistas y a una defensa mal comprendida de los asalariados. Habrá que trabajar en una transformación de los reglamentos y de las mentalidades apropiadas para hacer que, conservando su función de garantía última contra todo lo arbitrario, los títulos escolares sean tomados en cuenta por una duración limitada y jamás de manera exclusiva, es decir como una información entre otras.

Entre los factores apropiados para minimizar el efecto de estigmatización uno de los más eficaces será sin duda la instauración de nuevas formas de competencia. La competencia entre comunidades escolares asociando maestros y alumnos en proyectos comunes - tal como hoy en día se realiza en materia de deporte entre clases y establecimientos - tendrá por efecto suscitar una emulación, y en esta forma un estímulo al esfuerzo y a la disciplina que no tendrá por contraparte, como la competencia entre los individuos (alumnos y profesores), la atomización del grupo y la humillación o el desánimo de algunos.

\section{La unidad en el pluralismo y por el pluralismo}

La enseñanza deberá superar la oposición entre el liberalismo y el estatismo creando las condiciones de una emulación real entre instituciones autónomas y diversificadas, protegiendo a los individuos y a las instituciones más desfavorecidas contra la segregación escolar que pueda resultar de una competencia salvaje.

La existencia de una oferta escolar diversificada, propuesta a todos los niveles por instituciones de enseñanza autónomas y competitivas (a menos a nivel de la enseñanza superior), podría ser el principio de toda una serie de efectos convergentes apropiados para acrecer la eficacia y la equidad del sistema de enseñanza, reforzando la emulación entre los establecimientos, los equipos pedagógicos y las comunidades escolares y, de ese modo, favorecer la innovación y debilitar los efectos funestos de la condenación escolar. 
Los organismos de enseñanza deben ser colocados al abrigo de todas las presiones exteriores y dotados de una autonomía real, es decir, en los casos sobre todo de establecimientos de investigación, del poder de determinar ellos mismos sus objetivos. Entre las condiciones necesarias para asegurar a los establecimientos de enseñanza superior la autonomía, la especificidad y la responsabilidad que definen una verdadera universidad que conjuga la enseñanza fundamental y la enseñanza profesional, y dotada de recursos diversificados correspondientes a sus diversas funciones, la mas importante es sin duda el dominio de un presupuesto global que se podrá asegurar por medio de una pluralidad de fuentes de financiación: subvenciones del estado, de las regiones, de las municipalidades, de las fundaciones privadas, contratos con el Estado o las empresas públicas o privadas y quizá incluso participación financiera de estudiantes y antiguos alumnos. Establecimientos de enseñanza superior y de investigación con financiación privada o semi-pública podrían coexistir en esta forma con establecimientos de financiación exclusivamente pública. La autonomía deberá ser así completa en materia de creación de enseñanzas, de colación de grados y de regulación de flujo de estudiantes, debiendo sostener el Estado las enseñanzas no rentables económicamente pero culturalmente importantes.

De aplicación relativamente fácil en lo que concierne a la enseñanza superior, en que el proceso está ya comprometido -sin que hayan sido previstas las medidas capaces de contrarrestar los efectos de la competencia salvaje-, este principio podría encontrar una aplicación progresiva, a través de experiencias voluntarias poco a poco generalizadas, desde la enseñanza secundaria (de la que hoy en día habría que extender la definición hasta el nivel del DEUG incluido. NDLR: dos años después del bachillerato). Se trataría de crear establecimientos que ofrezcan, al lado de los saberes fundamentales universalmente exigibles, enseñanzas especiales optativas que constituirían su especialidad $\mathrm{y}$, de este modo, uno de sus puntos fuertes en la competencia. Esto supondría que los jefes de establecimientos o los colectivos de docentes dispongan de una autonomía más grande en el reclutamiento de maestros (por la introducción de criterios múltiples, entre los cuales habría criterios propiamente pedagógicos, y considerando la relación entre el perfil de los docentes así evaluados y el perfil de los empleos).

Se tenderá así a sustituir la competencia larvada por una emulación abierta, pero controlada y corregida gracias a una redefinición profunda del papel del Estado. Para contrarrestar el fortalecimiento que la selección por medio de los costos de escolaridad o la distancia geográfica podría aportar a la segregación escolar, el poder central deberá conferir a los individuos y a las instituciones más desprovistos garantías explícitas, eficaces y permanentemente repensadas contra los efectos de la competencia salvaje. Le deberá asegurar a todos las condiciones institucionales de adquisición del mínimo cultural común: otorgando becas de Estado, válidas en todos los establecimientos, a los estudiantes desprovistos de medios económicos para hacer valer sus capacidades escolares y dando a los establecimientos (universidades o liceos o colegios) los medios para asegurarles formas individuales o colectivas de tutoría; acordando subvenciones y ventajas institucionales (por ejemplo, primas para maestros) a los establecimientos que reciban a los más desfavorecidos, que en esta forma serían no depositarias de adolescentes condenados a la degradación, sino verdaderas zonas de educación prioritaria.

Respetando los particularismos culturales, lingüísticos y religiosos, el Estado debe asegurar a todos el mínimo cultural común que es la condición del ejercicio de una actividad profesional acertada y de la conservación del mínimo de comunicación 
indispensable al ejercicio ilustrado de los derechos del hombre y del ciudadano. En consecuencia, pertenecerá a los poderes públicos velar por la calidad pedagógica del conjunto de las instituciones de enseñanza fundamental (de los jardines infantiles a la tercera incluida).

El Estado deberá contribuir así directa e indirectamente a la formación de maestros, a la definición y evaluación de sus prácticas pedagógicas, especialmente a través de la revisión periódica de los programas, el otorgamiento de instrumentos pedagógicos de calidad (manuales), la definición del mínimo cultural común, pero también a través de acciones incitativas de orientación, como la oferta universal y gratuita de mensajes educativos de alta calidad que permitiría la creación de una cadena de televisión cultural.

Programas nacionales deberán definir el mínimo cultural común, es decir el núcleo de saberes y de habilidades fundamentales y obligatorias que deben poseer todos los ciudadanos. Esta formación elemental no debería concebirse como una especie de formación acabada y final sino como el punto de partida de una formación permanente. Deberá entonces poner el acento sobre los saberes fundamentales que son condición de adquisición de todos los demás saberes y sobre la disposición para adquirir saberes (adaptabilidad intelectual, apertura de espíritu, etc.). También deberá poner el acento sobre las formas de pensamiento y los métodos más generales y más ampliamente trasladables, como el dominio de la proporción o del razonamiento experimental. Todo deberá ser puesto en ejecución para dar a todos un dominio real de la lengua común, escrita y hablada -sobre todo en situación pública-. Sobre este último punto conviene no olvidar que para quienes no tienen al francés por lengua materna, esta adquisición supone sin duda otro acceso a un dominio real de su primera lengua, que condiciona el control de las diferencias fonéticas, gramaticales y estilísticas. Sería muy deseable que también fuese enseñada lo más pronto posible una lengua extranjera.

En esta perspectiva, el jardín infantil, que en todas partes debería obtener los medios de recibir a los niños desde la edad de tres años, podría, al menos en su fase final, asociar a la enseñanza de expresión una enseñanza de formación tendiente a transmitir los saberes fundamentales de los que la escuela primaria presupone tácitamente el dominio, comenzando por la comprensión y el uso de la lengua común y de diversas técnicas verbales y gráficas.

Con la intención de reforzar o de restaurar la motivación de los maestros y de contrarrestar la disolución de las responsabilidades que promueven el recurso a la simple antigüedad como único patrón de todas las formas de competencia, el poder de tutela debería instituir instancias de evaluación de la actividad pedagógica y científica de los maestros. Estas instancias designadas para un período limitado (máximo cinco años), compuestas al menos en parte de personas extrañas al cuerpo considerado y escogidas por sus contribuciones creadoras en las más diversas actividades, tendrán que evaluar la calidad de los individuos y de las instituciones (equipos educativos, establecimientos, etc.) en función de criterios múltiples y matizados; el peso de los títulos por ejemplo deberá ser contrabalanceado o suplantado por la consideración de la efectividad pedagógica que, dándose las disparidades de reclutamiento social de los alumnos según el establecimiento, no puede medirse con el único índice del éxito en los exámenes. Podrán así orientar la atribución a las instituciones y a los maestros de ventajas materiales y simbólicas (subvenciones, promociones, primas, estancias de formación en el extranjero, viajes de estudio, etc.). 
Estas instancias de evaluación podrán cumplir las mismas funciones a nivel de enseñanza secundaria. Una vez realmente instauradas las condiciones de la competencia entre los establecimientos, en esta forma incitados a vincular los mejores, sus evaluaciones podrán guiar la selección de los padres de familia y de los alumnos. A estas instancias permanentes se podrán agregar misiones temporales confiadas a especialistas (franceses y extranjeros) y destinadas a describir de la manera más precisa, referente a lo que existe y a lo que cambia en Francia y en el extranjero, el estado científico y pedagógico de las personas y de los establecimientos de diferentes niveles en la especialidad considerada. La atribución de una ayuda pública tendrá de hecho que ser subordinada a la aceptación de someter-se a esos controles incitativos, apropiados para asegurar a los establecimientos correspondientes testimonios de calidad y, de ese modo, una ventaja en la competencia.

Debería hacerse todo para aumentar la parte de autonomía y de responsabilidad de los docentes o de los equipos pedagógicos en el ejercicio de su oficio, vinculando los docentes a la producción de instrumentos de gran difusión (video-casetes, manuales, etc.), a la elaboración de técnicas pedagógicas, a la revisión de contenidos. Lo que supondrá la creación de equipos de animadores itinerantes encargados de recibir las críticas y las sugerencias a propósito de instrumentos y métodos en uso, de asistir técnica y financieramente a quienes innovan, y de hacer circular la información sobre todas las iniciativas de progreso, brevemente dicho, de descubrir, animar y ayudar en todas las formas posibles a los maestros de mayor invención. Estos animadores tendrán después por función organizar periódicamente a escala regional, es decir en unión con universidades locales, reuniones de trabajo en las que los maestros podrán plantear problemas prácticos de su enseñanza delante de especialistas que obran no como una instancia de control y de inspección sino como institución de consejo y asistencia.

\section{La revisión periódica de los saberes enseñados}

El contenido de la enseñanza deberá ser sometido a una revisión periódica tendiente a modernizar los saberes enseñados, eliminando los sabe-res caducos o secundarios o introduciendo lo más rápidamente posible las nuevas adquisiciones, sin ceder al modernismo a ultranza.

La inercia estructural del sistema de enseñanza que se traduce por un retardo, más o menos grande según los momentos y según los dominios, de los contenidos enseñados en relación con las adquisiciones de la investigación y a las demandas de la sociedad, debería ser metódicamente corregida por medio de intervenciones reglamentarias o incitaciones indirectas encaminadas a favorecer la revisión de los programas, de los manuales, de los métodos e instrumentos pedagógicos. Para ser aceptables y aplicables, estas revisiones evidentemente deberán tener en cuenta de la manera más realista las obligaciones y los límites propios de la institución escolar.

El crecimiento continuo de los saberes impuestos tiende por una parte a los efectos de la inercia institucional y mental que conduce a acompañarse indefinidamente de lo que una vez existió. Es así corno el corporativismo de disciplina conduce a perpetuar saberes caducos o superados, y divisiones que pueden persistir en el organigrama escolar, a través de los exámenes, de los concursos, de los diplomas, de los manuales y de la enseñanza, cuando ya no tienen vigencia en el orden de la investigación. Otro factor de conservación, la tendencia al enciclopedismo, la pretensión de exhaustividad o la institución de los datos previos por los requisitos absolutos ("no se puede ignorar eso"). Para combatir esas tendencias, y los corporativismos que las fundamentan, habría que 
reunir un comité de revisión de programas (de las enseñanzas primaria y secundaria) encargado de podar los programas de todas las exigencias vestigiales y de introducir lo más rápidamente posible innovaciones útiles. Esto sin ceder al modernismo a ultranza; sobre todo cuando, como es el caso, por ejemplo, en historia, en que no corresponde a ningún cambio decisivo, la modificación inconsiderada de los programas tiene por efecto introducir repeticiones y lagunas en el decurso de sus estudios de los estudiantes. Ciertamente hay que iniciar a los alumnos en las grandes revoluciones conceptuales sobre las que reposa la ciencia moderna, pero hay que evitar hacerlo demasiado temprano y sobre todo en detrimento de una ciencia clásica, a menudo muy formadora, sobre la que reposa todo el edificio: pretender enseñar la relatividad restringida a principiantes que no saben cómo funciona un transformador no tiene casi sentido. Tal comité de revisión deberá tener poder para orientar la política de inversiones públicas en materia de educación, definiendo los sectores que hay que reducir o aumentar, y orientando la elaboración y la ejecución de instrumentos pedagógicos nuevos.

\section{La unificación de los saberes transmitidos}

Todos los establecimientos escolares deberán suponer un conjunto de conocimientos considerados necesarios en cada nivel, cuyo principio unificador podría ser la unidad histórica.

Para compensar los efectos de la especialización creciente que condena a la mayoría de los individuos a saberes parcelarios, y en especial la escinsión, cada vez más marcada, entre los "literarios" y los "científicos", hay que luchar contra la insularización de los saberes ligada a la división en disciplinas yuxtapuestas; se trata por esto de elaborar y difundir, a lo largo de la enseñanza secundaria, una cultura que integre la cultura científica y la cultura histórica, es decir, no sólo historia de la literatura o incluso de las artes y de la filosofía, sino también historia de las ciencias y de las técnicas; la misma tendencia a la insularización se observa en el seno de un mismo sector de la cultura y hay que animar por ejemplo la progresión coordenada de las enseñanzas científicas, especialmente de las matemáticas y de la física.

Uno de los principios unificadores de la cultura y de la enseñanza podría ser así la historia social de las obras culturales (de las ciencias, de la filosofía, del derecho, de las artes, de la literatura, etc.), uniendo de manera a la vez lógica e histórica el conjunto de adquisiciones culturales y científicas (por ejemplo la historia de la pintura del Renacimiento y el desarrollo de la perspectiva matemática). La reintegración de la ciencia y de su historia en la cultura de donde está de hecho excluida, tendrá por efecto favorecer a la vez una mejor comprensión científica del movimiento histórico y una mejor comprensión de la ciencia que, para comprenderse mejor ella misma, tiene necesidad de un conocimiento racional de su historia, y que revela mejor la verdad de su recorrido y de sus principios cuando es aprehendida en las incertidumbres y las dificultades de sus comienzos. La enseñanza de una visión más histórica de la ciencia tendrá por efecto favorecer una representación menos dogmática tanto de la ciencia como de su enseñanza, y de llevar a los maestros de todos los niveles a poner en primer plano los problemas tanto como las soluciones, y recordar, en cada caso, los que han sido los programas de investigación concurrentes.

La historia de las obras culturales (ciencia, arte, literatura, etc.) debería ser enseñada en su dimensión internacional, en especial europea; en consecuencia, los docentes de la lengua y de la literatura nacionales y de las lenguas y las literaturas extranjeras, deberán estar estrechamente asociados, sin privilegio jerárquico. Para conciliar los imperativos de 
universalismo y las funciones de integración cultural de toda enseñanza de cultura, importará propiciar y favorecer la redacción de manuales de historia de la civilización y de obras culturales del mundo europeo, así como de otros grandes conjuntos culturales, reuniendo representantes eminentes, franceses y extranjeros, de diferentes disciplinas y traducir el resultado de estos trabajos a medios de transmisión como el video-casette.

Una verdadera universidad abierta con vocación europea, concebida sobre un modelo inspirado en la Open University inglesa, podrá ofrecer una enseñanza televisada abastecida de documentos de acompañamiento y de un recuadro (correcciones de ejercicios, aclaraciones complementarias, etc.) que podría asegurarse, en el marco regional, por una antena universitaria; una de las funciones de esta institución sería la de procurar a los profesores de todos los niveles un instrumento cómodo de formación continua y, si llegase el caso, de promoción, lo que tendría por efecto incitar inversiones propias para favorecer la conservación o el mejoramiento de la competencia de los maestros. Se podría incluso concebir que apoyándose en un satélite europeo y a costa de una firme simplificación de equivalencia, esta universidad abierta llegue a difundir a escala europea enseñanzas multilingües de alto nivel y ofrecer una preparación para exámenes ordinarios de enseñanza superior, contribuyendo así a una unificación europea de enseñanzas y de títulos.

\section{Una educación ininterrumpida y alternada}

La educación se deberá proseguir a lo largo de la vida, y se deberá hacer todo lo posible para reducir la ruptura entre el fin de la enseñanza y el ingreso a la vida activa.

La institución escolar es uno de los factores de la diferenciación en clases de edad. Habría que trabajar por hacer que no haya edad de ir a la escuela, que en todo caso no haya límites superiores. Lo que supondría que sea abierta la posibilidad de escolarización en todos los niveles para todas las edades al precio de una transformación de las representaciones que conducen a asociar un cierto nivel de competencia con cierta edad. $\mathrm{Si}$ se sabe que las diferencias sociales se reducen muy rápidamente en diferencias de edad escolar (adelanto, "precocidad" o retardo, etc.), se ve que un acrecentamiento de la elasticidad de la edad escolar podría tener efectos sociales importantes.

Si de hecho se observa la aparición de una clase de edad de trabajadores-estudiantes y de estudiantes-trabajadores, la combinación del trabajo y de los estudios no ha encontrado verdaderamente su sanción en las instituciones. Sin duda porque esto supondría que sean repensadas completamente la noción misma de trabajo y la oposición entre la inactividad y el trabajo, dentro del cual habría que incluir actividad profesional y formación. Por razones inseparablemente técnicas y sociológicas, la formación debería considerarse, especialmente en el caso de los docentes, como un trabajo completamente aparte, que confiere un verdadero status social y que arranca al mismo tiempo a los estudiantes-trabajadores del estado de indeterminación social en que hoy en día están, lo que será tanto más fácil cuanto la ruptura entre teoría y práctica haya sido debilitada. El ejercicio de una actividad profesional podrá a menudo ser conducida al mismo rango con la continuación de los estudios superiores en lugar de ser expulsado más allá del fin de los estudios. En esta perspectiva, se podría concebir la enseñanza superior como una institución de educación permanente que pone en ejecución todos los medios disponibles, escolares y extraescolares (radio, televisión, video, etc.) y apropiada para hacer desaparecer la ruptura, tanto más brutal e irreversible cuanto más precoz sea, entre los estudios y la vida profesional. Más ampliamente, todas las ocasiones, y en especial el 
servicio nacional, deberían ser aprovechadas para dar nuevas oportunidades de formación a quienes están ya trabajando.

Semejante objetivo no puede alcanzarse más que a costa de una profunda transformación de la organización escolar y de las mentalidades. En efecto, para que el derecho de recibir una formación en cualquier edad llegue a ser real, habrá que dejar de identificar la formación y la escolarización, creando por ejemplo instituciones de formación apoyadas en la televisión, sancionando la formación adquirida por el trabajo o por el esfuerzo personal, etc. Habría también que repensar profundamente la organización escolar de manera que permita que los periódicos retornos a la escuela puedan tener múltiples duraciones (por un año, con el modelo de año sabático, pero también por seis o tres meses, o una semana, o por dos horas diarias —en el curso de la tarde_, por ejemplo) y tomar formas muy diversas: ciclos de formación, estadios intensivos, etc. Podrían instituirse fórmulas de intercambio entre instituciones escolares y las empresas públicas o privadas, en particular bajo la forma de estadios de formación o de perfeccionamiento (en los niveles elevados del curso esos intercambios serían sin duda extremadamente provechosos a ambas partes), y recíprocamente, por períodos de formación o reciclaje, en la universidad. Las fórmulas de alternar el estudio con la escuela y el trabajo con la empresa o el laboratorio debería generalizarse, dando así su pleno sentido a la educación permanente.

\section{El uso de las técnicas modernas de difusión}

La acción de incitación, de orientación y de asistencia del Estado de be-ría ejercerse por medio de un uso intensivo y metódico de técnicas modernas de difusión de la cultura, y en especial de la televisión y de la telemática, que permitirán ofrecer a todos y en todas partes una enseñanza ejemplar.

La sabia utilización de los instrumentos modernos de comunicación, y especialmente de la videocaseta, permite hoy en día confiar la producción de instrumentos de transmisión de saberes y habilidades elementales, es decir fundamentales, a equipos que asocien especialistas de la comunicación audiovisual, capaces de emplear lo mejor posible las capacidades específicas del instrumento, y profesores designados por sus competencias pedagógicas particulares que evidentemente tendrían interés en rodearse de la opinión de la comunidad científica. A diferencia de la enseñanza televisada que debido a la rigidez de la programación, se integra a menudo mal en la progresión, necesariamente diversa, de la enseñanza en los establecimientos escolares, el video permite ofrecer enseñanzas cortas, densas y pedagógicamente eficaces: de un cuarto de hora o máximo de media hora, dejando el lugar al comentario, a la discusión y al ejercicio. Dirigiéndose a saberes y puntos del programa en que la imagen (fotografía, animación, etc.) es irremplazable, esas enseñanzas deberían ser definidas, para cada nivel de enseñanza y cada dominio del saber, en pliegos de peticiones destinados a los productores, públicos y privados, de videocasetas susceptibles de ser utilizadas en los establecimientos escolares. La videocaseta, que permite combinar la agilidad de la utilización, pudiendo cada maestro de cada establecimiento escoger el momento para utilizarla, y la unidad de concepción y de realización, podrá contribuir a unir la libertad y la diversidad de los usos pedagógicos y la unidad y la cualidad de la cultura enseñada. El uso razonado y generalizado de enseñanzas apreciadas como de alto nivel contribuirán por añadidura a reducir el efecto de las variaciones de calidad de las enseñanzas según los establecimientos, las regiones, etc. No es dudoso, por ejemplo, que en materia de arte y de literatura, y muy especialmente de teatro, y también de geografía y de lenguas vivas, la imagen podría contribuir a quitar a la enseñanza el carácter demasiado irreal que reviste para los niños o los 
adolescentes desprovistos de experiencia directa del espectáculo o del viaje al extranjero. Para favorecer la producción de esos nuevos instrumentos pedagógicos, habrá que suministrar a los docentes interesados los medios de adquirir las nuevas técnicas de transmisión del saber, y a quienes las hayan dominado perfectamente, los medios de ponerlas en ejecución.

La televisión podría ser utilizada, especialmente el sábado y e] domingo, como base de una verdadera educación permanente (a diferentes niveles) que satisfaría una demanda actualmente explotada por los mercaderes de cursos por correspondencia, de enciclopedias y de otras obras y revistas de vulgarización, y crearía, en torno a la escuela, un entorno cultural indispensable al éxito generalizado de la empresa educativa.

Una combinación razonada de la videocaseta y de la telemática podría permitir a establecimientos de enseñanza equipados de terminales de ordenador proponer una educación personalizada de alto nivel. Vemos como, por este medio, la ayuda del Estado, bien distribuida, podría compensar las desventajas geográficas y sociales. Se tratará, en un primer momento, de lanzar un pequeño número de experiencias a la vez sociológicamente válidas (es decir, aseguradas de todas las condiciones necesarias para tener oportunidades de éxito) y susceptibles de ser reproducidas a una escala mayor después de sacar sus conclusiones y encontrado los medios.

Para evitar las ilusiones y sobre todo las desilusiones, hay sin embargo, que recordar que los instrumentos modernos de enseñanza no pueden ser eficaces más que a condición de que no se les pida que sustituyan a los maestros sino que los asistan en una tarea renovada por su utilización; jamás son más que un útil suplementario a disposición de los maestros cuya competencia, brillo y entusiasmo continúan siendo los factores principales del éxito pedagógico. Por añadidura, no pueden recibir toda su eficacia más que a precio de enormes inversiones económicas y culturales. El acceso al "consumo individual" de la educación que permite el recurso de la telemática tiene por efecto aumentar los costos de la educación y no de reducirlos como se ha podido creer. No solamente porque exige gastos importantes en equipos (televisores, magnetófonos, microordenadores o terminales de ordenador, videotecas, etc.) que al favorecer una pedagogía activa, y un trabajo individual o colectivo de investigación, son ellos mismos generadores de nuevas necesidades (bibliotecas de investigación, bancos de datos, etc.); pero también porque demanda maestros muy competentes y muy comprometidos en una empresa pedagógica apropiada para revelar nuevas necesidades pedagógicas — siendo propia la utilización imperfecta, por el contrario, para determinar regresiones en relación con lo que permitían alcanzar los medios tradiciona1es El efecto de homogenización y centralización que produciría el uso sistemático (pero necesariamente limitado a una fracción restringida del horario) de enseñanzas fabricadas a nivel de instancias centrales, tendría por contraparte la acción diversificada de los maestros: lejos de encontrarse reducidos al papel de simples repetidores, tendrán que cumplir una función completamente nueva, desembarazada de tareas puramente repetitivas y combinando la acción continua y personalizada cual es la del tutor, encargado de acompañar al aprendiz en su trabajo, y la acción pedagógica del maestro y del animador, destinados a transmitir los modos de pensamiento más fundamentales, y a organizar el trabajo individual o colectivo.

Un cambio de vehículo exige cambios en el mensaje. Los medios de comunicación modernos no pueden obtener su pleno rendimiento más que a condición de que se opere una profunda transformación de los contenidos enseñados y de las maneras de enseñar: se trata en cada caso de hacer un esfuerzo metódico para lograr el máximo de 
rendimiento de la comunicación (por medio de un trabajo sobre el lenguaje empleado, sobre los documentos presentados, las experiencias propuestas), tomando en cuenta explícitamente las características sociales y escolares de los destinatarios. Para responder a la demanda de formación y de información que la utilización de esos nuevos medios no podría dejar de hacer surgir, la autoridad central debería apoyarse en equipos de animadores que deberán permitir encontrar, en un conocimiento crítico de las mejores soluciones aportadas por algunos a los problemas con que todos se han enfrentado, las bases de una generalización progresiva y voluntaria de experiencias atinadas.

\section{Apertura en la autonomía y por la autonomía}

Los establecimientos escolares deberán asociar a sus deliberaciones y a sus actividades personas externas, coordenar su acción con la de otras instituciones de difusión cultural y llegar a ser el centro de una nueva vida asociativa, lugar de ejercicio práctico de una verdadera instrucción cívica; paralelamente, habrá que reforzar la autonomía del cuerpo docente revalorizando la función profesoral y reforzando la competencia de los maestros.

Sin ceder al mito de la "apertura a la vida", que puede conducir a aberraciones, y sin comprometer la autonomía indispensable de la institución escolar con respecto a la demanda social, hay que evitar que el sistema escolar se constituya en universo separado, sagrado, proponiendo una cultura también sagrada y liberada de la existencia ordinaria. Por esto sería indispensable que todas las instituciones de transmisión cultural (escuelas, museos, bibliotecas, etc.) asocien en sus consejos, de manera mucho más real y más eficaz que hoy en día, a personalidades exteriores (lo que no quiere decir notables), no dentro de la lógica de un control que no puede suscitar sino reacciones de veto y de defensa corporativista, sino de la lógica de participación en las responsabilidades, incluso financieras, en la inspiración y en la incitación.

La escuela no puede y no debe ser el único lugar de formación; no puede y no debe pretender enseñarlo todo. La transmisión de los saberes no pudiendo, ni de hecho ni de derecho, ser monopolizada por una única institución, tendrá que tomar en cuenta la red de lugares de formación complementarios dentro de la cual debe definirse la función específica de la escuela. Debido a la importancia creciente de las acciones de difusión cultural que se ejercen por fuera de la institución escolar — por medio de la televisión sin duda, pero también del teatro, del cine, los hogares juveniles y las casas de la cultura, etc.-, la acción escolar podrá tener su rendimiento intensificado si se integra consciente y metódicamente en el universo de acciones culturales ejercidas por los otros medios de difusión. Habría que buscar, tanto a escala nacional como a escala de las pequeñas unidades locales, la articulación de todas las formas de difusión cultural, y favorecer, al menos a escala de los pequeños pueblos, la coordinación de la acción escolar con la de las diferentes instituciones de difusión cultural: bibliotecas, museos, orquestas, etc., y también de diferentes agentes de producción y de difusión culturales: profesores, artistas, escritores, investigadores. Importaría por esto superar los obstáculos técnicos, financieros, y sobre todo burocráticos o jurídicos (especialmente en materia de responsabilidad civil), sin hablar de resistencias psicológicas, que hoy en día frenan esos cambios, e impiden especialmente la participación, voluntaria o remunerada, en la enseñanza de personalidades de los mundos artístico, científico o profesional. Necesaria a nivel de la producción de obras culturales, la ruptura institucional, pero también mental, entre productores de cultura -investigadores, artistas, escritores- y transmisores de cultura - profesores, periodistas, editores, directores de galería, etc.- debería reducirse a nivel pedagógico. La entrada en el universo escolar de verdaderos creadores de cultura, 
siempre llamados y acogidos por los docentes que obran de mediadores encargados de preparar y prolongar sus intervenciones puntuales, tendrá por efecto recordar la distinción, sin duda parcialmente irreductible, entre cultura y cultura escolar.

La función propia de la acción escolar y de la selección de contenidos enseñados deben ser repensados por referencia al conjunto de las instituciones de difusión complementarias y concurrentes: esto con el fin de evitar los dobles empleos y para concentrar el esfuerzo pedagógico en los terrenos en que es irremplazable la acción de la escuela. De hecho la escuela debe prioritariamente dirigir su esfuerzo a la inculcación de las disposiciones generales y transportables, que no se pueden adquirir más que por la repetición y el ejercicio. Ella sola puede en efecto transmitir los útiles de pensamiento que condicionan la comprensión de todos los mensajes y la integración racional de todos los conocimientos al mismo tiempo que la síntesis crítica de los saberes susceptibles de ser adquiridos por otras vías, saberes o semisaberes a menudo dispersos, incluso fragmentados, como las condiciones, más o menos aleatorias, de su adquisición.

Los profesores deberían estar preparados y animados para utilizar, de manera reflexiva y crítica, los mensajes culturales suministrados por la televisión, el teatro, el cine, los periódicos. Por ejemplo, deberían recibir, en cualquier momento de su formación, una enseñanza sobre la técnica de los nuevos medios de comunicación que los hará aptos para transmitir la conciencia y el conocimiento de los procedimientos y los efectos (en especial de montaje y de imagen) que, por el tiempo que permanezcan ignorados, confieren a mensajes e imágenes socialmente construidos, las apariencias de lo natural y de la evidencia.

Todos los esfuerzos apropiados para favorecer la construcción de una verdadera comunidad educativa que una en un intercambio de información o de servicios a padres de familia y educadores (lo que es el caso a menudo en los establecimientos privados) y deberían ser animados -al mismo tiempo que sería claramente delimitado el dominio propio de la competencia profesoral. La escuela abierta debería llegar a ser una especie de casa común, centro de encuentro entre las generaciones y entre las clases sociales, en especial entre los antiguos residentes y los nuevos inmigrados, en favor, por ejemplo, de cursos de alfabetización. Sería un lugar en el que podría aprenderse y ejercerse, sobre todo en las pequeñas aglomeraciones, con ocasión de acciones prácticas de ayuda mutua o de auxilio, con respecto o particular de personas mayores o de minusválidos o a través de las actividades asociativas que unan generaciones diferentes (actividades artísticas, deportivas, clubes de recreación, etc.), una moral de la responsabilidad, de la solidaridad y del respeto a los demás: la educación cívica encontrará allí sus trabajos prácticos. Estas actividades colectivas serán en efecto la ocasión de inculcar cierto número de principios de la existencia social: en particular que la vida colectiva, y singularmente aquella de una clase activa y orientada a un proyecto común, es imposible sin ciertas restricciones que cada uno debe imponerse; que no se puede trabajar en una misma actividad sin una disciplina mínima...

Muchas actividades de interés general que han sido delegadas a los poderes públicos, trátese de actividades propiamente culturales, como las exposiciones, los espectáculos, los conciertos, etc., o de aquellas que hacen referencia, por ejemplo, a la belleza de los lugares públicos, a la preservación del entorno natural, al respeto a los animales, etc., podrían ser organizadas en las instituciones escolares, a través suyo o en torno a ellas, devolviendo a los educadores el papel social de caudillos ejemplares que a menudo les correspondió. 
Correlativamente a este esfuerzo para ofrecer a los maestros una función enriquecida y ampliada y, de ese modo, más atrayente, y para revalorizar socialmente esta función dando a la escuela los medios de jugar un papel central en la vida social, también se podría prever la formación permanente que es necesaria para asegurar la conservación y la renovación de la competencia técnica y pedagógica en un universo en que los saberes evolucionan rápido, por el hecho de que la investigación científica y el desarrollo tecnológico, y en el que las especialidades se reestructuran y se diversifican: el trabajo individual de ponerse al día debería ser el resultado de ventajas inmediatas, y podría favorecerse por medio de una utilización sistemática de la enseñanza televisada (universidad abierta).

El oficio de enseñar es un oficio difícil, a veces penoso y agotador, que no puede realmente ser exaltador y eficaz si no se ejerce con pasión y convicción. Los maestros de todos los órdenes de enseñanza no pueden escapar al desgaste psicológico y técnico más que a condición de que puedan romper periódicamente con la rutina escolar saliendo del universo cerrado de la escuela para hacer períodos de práctica en laboratorios, empresas, etc.; o para retomar su formación sea por medio de un trabajo personal sea para continuar estudios aprovechando los años sabáticos. $Y$ será necesario sin duda ofrecer a los maestros de cierta edad que lo desearen la posibilidad de completar su carrera en funciones de administración, en tareas culturales menos pesadas (como las actividades de tutor o de animador itinerante), según sus preferencias y sus aptitudes.

Una fuerte competencia específica, tanto en lo que concierne a la materia enseñada como en lo referente a la manera de enseñar, constituye sin duda el mejor, sino el único garante, de la autonomía de la escuela y de la independencia de los maestros con respecto a todos los grupos de presión.

\section{De la aplicación de los principios}

Estas proposiciones se inspiran sin duda en una posición optimista que se observa en particular en el esfuerzo por superar las contradicciones entre los objetivos opuestos que el sistema escolar debe perseguir. ¿Pero cómo no tener en mente los apremios con que debe contar toda acción sobre el funcionamiento de la escuela? ¿Y cómo olvidar que un discurso bien intencionado no puede bastar para producir el mejor de los mundos escolares posibles? Muchas veces en el pasado, medidas inspiradas en las mejores intenciones han arrojado resultados que iban a la inversa de los fines propuestos. $Y$ cuando se está obligado, por ejemplo, a recordar las virtudes del aprendizaje por la experiencia, incansablemente exaltadas, desde Rosseau y Pestalozzi, por todos los reformadores, no se puede escapar al sentimiento de que la institución es capaz de neutralizar o de desviar todas las medidas destinadas a transformarla. Así, ¿cómo serán escogidos quienes tendrán la misión de elegir las innovaciones reales y de recompensar los verdaderos méritos? ¿Cómo serán descubiertos y animados los responsables capaces de movilizar, por su competencia y su dinamismo, las inmensas reservas de imaginación y de consagración aún mal utilizadas? ¿Cómo superar las resistencias innumerables que podrán encontrar justificaciones en las dificultades técnicas del oficio de docente, a menudo ignoradas por el gran público, y en las condiciones materiales a menudo deplorables en las cuales debe ejercer? ¿Cómo desbaratar las artimañas del formalismo igualitarista que permite evitar reconocer la evidente desigualdad de las condiciones de formación, y confiar las tareas más difíciles, sin preparación particular y sin contrapartida especial, a los maestros más jóvenes y más desprovistos? 
La importancia de estos puntos de partida, asociados a la educación, impone poner en ejecución todo para superar estos obstáculos sociales. No se puede diferir por más tiempo el esfuerzo necesario para mejorar las condiciones de existencia, de formación y de trabajo de los maestros a todos los niveles, y la instauración de medidas reglamentarias indispensables para recompensar sus éxitos. No se puede hacer por más tiempo la economía de importantes inversiones en la infraestructura propiamente cultural (comenzando por las bibliotecas).

Paralelamente habría que emprender sin demora cierto número de experiencias limitadas pero decisivas: universidad abierta, comité de revisión y de unificación de los programas, institutos regionales de formación continuada de maestros (trabajando en unión con la universidad abierta), unidades experimentales de enseñanza personalizada utilizando la videocaseta y la telemática, cuerpo de animadores, comité ejércitouniversidad para la organización de la formación, cadena de televisión cultural, etc. Con el fin de evitar que vengan a reforzar por el fracaso o la apariencia de éxito los mecanismos mismos que pretenden combatir, esas experiencias no deben emprenderse más que a condición de que se encuentren reunidas todas las condiciones materiales e intelectuales indispensables para su éxito y que sean reproducibles y generalizables.

Enseñar no es una actividad como las otras: pocos oficios pueden ser causas de riesgos más graves que aquellos que los malos maestros hacen incurrir a los alumnos que le son confiados; pocos oficios suponen tantas virtudes de generosidad, de consagración y, sobre todo, quizá de entusiasmo y de desinterés. Sólo una política inspirada por el deseo de atraer y promover a los mejores, esos hombres y esas mujeres de calidad que todos los sistemas de educación siempre han celebrado, podrá hacer del oficio de educador de la juventud lo que debería ser, el primero de los oficios. 